\title{
Contextos de vulnerabilidade entre adolescentes do ensino fundamental de Uberaba/MG
}

Recebido em: 13/12/2011

Aceito em: 27/09/2012
Rodrigo Eurípedes da Silveira ${ }^{1}$ Álvaro da Silva Santos ${ }^{2}$

Estudo ecológico-exploratório, que objetivou descrever situações de vulnerabilidade entre 189 alunos de $7^{\mathrm{a}} \mathrm{S}$ e $8^{\mathrm{a}} \mathrm{s}$ séries de uma escola pública de Uberaba/ MG. A iniciação sexual já havia ocorrido para $66,7 \%$ dos jovens e $40,5 \%$ não utilizavam métodos contraceptivos. O uso de álcool foi relatado por $66 \%$ dos garotos e $55,1 \%$ das meninas; e de drogas ilícitas, 38\% e 25,8\%, respectivamente. Tais resultados refletem a necessidade de ações de educação em saúde direcionada a adolescentes, com metodologias mais inclusivas e lúdicas, estimulando a reflexão crítica acerca de fatores de vulnerabilidade relacionados ao comportamento sexual, uso de bebidas alcóolicas e drogadição.

Descritores: Adolescente, Vulnerabilidade, Educação em Saúde, Saúde Escolar.

\section{Contexts of vulnerability among adolescents of the elementary school of Uberaba/MG}

Ecological and exploratory study, which aimed to describe situations of vulnerability among 189 students in 7th and 8th series of a public school in Uberaba/MG. The sexual initiation had already occurred to $66.7 \%$ and $40.5 \%$ did not use contraceptive methods. Alcohol use was reported by $66 \%$ of boys and $55.1 \%$ of girls; and illicit drugs, $38 \%$ and $25.8 \%$, respectively. These results reflect the need of health education activities targeted at adolescents, more inclusive and methodologies with playful, stimulating the critical reflection about vulnerability factors related to sexual behavior, use of alcohol and drug addiction

Descriptors: Adolescent, Vulnerability, Health Education, Scholar Health.

\section{Contextos de vulnerabilidad entre los adolescentes de la escuela primaria de Uberaba/MG}

Estudio ecológico y exploratorio, cuyo objetivo fue describir situaciones de vulnerabilidad entre 189 estudiantes de 7 ○ y 8 o serie de una escuela pública en Uberaba/MG. La iniciación sexual ya había ocurrido a $66,7 \%$ de los jóvenes y $40,5 \%$ no usaban anticonceptivos. Lo consumo de alcohol fue reportado por $66 \%$ de los hombres y 55.1\% de las niñas; y de drogas ilícitas, por 38\% y 25,8\%, respectivamente. Estos resultados reflejan la necesidad de actividades de educación en Salud, más inclusivos y con metodologías lúdicas dirigidas a adolescentes, estimulando la reflexión crítica sobre factores de vulnerabilidad relacionados a comportamiento sexual, uso de alcohol y drogadicción.

Descriptores: Adolescente, Vulnerabilidad, Educación en Salud, Salud en la Escuela.

\section{INTRODUÇÃO}

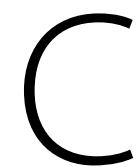

ada vez mais os pesquisadores das áreas de ciências sociais e de saúde têm se preocupado com as questões multidimensionais inerentes ao período da adolescência, em que o indivíduo deve lidar com a afirmação de sua identidade de gênero e suas funções, erotismo, prazer, intimidade e reprodução. Trata-se de uma fase perpetrada por muitas incertezas, que compõem um quadro de profundas alterações biológicas, psicológicas, sociais e outras, em que o adolescente passa a expressar sua busca por autonomia ${ }^{(1)}$.

É a partir da década de 1970 que a gestação na adolescência ganha visibilidade como problema de saúde no Brasil, dadas as crescentes taxas de natalidade entre garotas de 10 a 19 anos de idade $^{(2)}$. Nos dias atuais, os aspectos referentes à sexualidade na adolescência têm inspirado investigações nas cinco regiões do País, como expressam os estudos realizados nos estados de Mato Grosso(3), Rio de Janeiro ${ }^{(4)}$, Ceará(5), Santa Catarina ${ }^{(6)}$ e Amazonas ${ }^{(7)}$, haja visto que muitas vezes, o adolescente se sujeita a práticas sexuais desprotegidas.
De acordo com o Censo Demográfico de 2010, os adolescentes representam 17,9\% da população brasileira, tendo concentrado, em dez anos, 26\% das internações relacionadas a gravidez, parto e puerpério na Rede do Sistema Único de Saúde (SUS), com um gasto acumulado acima de $\mathrm{R} \$ 2$ bilhões ${ }^{(8)}$. Em Uberaba/MG, os adolescentes representam cerca de $16 \%$ da população do município, valor que chega a 53\% no Bairro Residencial 2000, onde $30 \%$ das jovens nessa faixa etária eram mães ou estavam grávidas ${ }^{(1)}$.

Contudo, a gestação na adolescência não condensa o problema em si, e sim através das iniquidades sociais a ela relacionadas. Aqui se insere o contexto de vulnerabilidade, cujos componentes (individual, social e institucional) são focos das ações da Política de Saúde dos Adolescentes e Jovens, que devem ser organizadas em rede de atenção à saúde, intra e intersetorialmente, pela análise da situação de um território e pela participação social|(9).

Alinhado a essas perspectivas, o Projeto de Extensão com interface à Pesquisa - "O Protagonismo Juvenil como Estratégia 
de Intervenção Familiar e Comunitária no Residencial 2000" teve suas ações direcionadas a pais/responsáveis, professores e alunos de $7^{\mathrm{a}}$ e $8^{\mathrm{a}}$ séries de uma escola pública municipal de Uberaba/MG, a partir de uma avaliação das situações de vulnerabilidade às quais aqueles jovens estavam inseridos.

Dessa forma, o presente estudo apresenta os resultados da investigação que precedeu as ações de extensão, tendo como objetivo descrever o perfil sociodemográfico e a presença de situações de vulnerabilidade, no tocante à atividade sexual e ao uso de drogas lícitas e ilícitas.

\section{MATERIAL E MÉTODO}

Estudo ecológico e exploratório, de corte transversal, cuja amostra incluiu alunos das $7^{\mathrm{a}} \mathrm{s}$ e $8^{\mathrm{a}} \mathrm{s}$ séries da Escola Municipal Esther Limírio Brigagão, em Uberaba/MG. Dos 203 estudantes, 189 foram incluídos na pesquisa, tendo devolvido o Termo de Consentimento assinado por seus responsáveis, uma vez que eram menores de 18 anos.

A coleta de dados ocorreu entre setembro de 2010 e março de 2011, através de um questionário semiestruturado, previamente ajustado por teste piloto em escola diferente daquela que foi objeto da pesquisa, sendo aplicado com a supervisão dos pesquisadores.

As variáveis estudadas foram: sexo, idade, renda familiar, iniciação sexual e utilização de métodos anticoncepcionais, uso de drogas ilícitas e bebidas alcoólicas. Os dados foram analisados no programa Epi-Info, versão 7.0 por meio de frequências absolutas e relativas. Considerou-se o valor de $p<0,05$.

A presente investigação foi submetida ao Comitê de Ética em Pesquisa com Seres Humanos da Universidade Federal do Triângulo Mineiro e aprovada pelo protocolo n¹.764/2011.

\section{RESULTADOS}

A população masculina predominou entre os alunos de $7^{a}$ série em $53,6 \%$ e em $52,2 \%$ na $8^{a}$ série. A idade média da população estudada foi de $14,6 \pm 1,3$ anos. $50,3 \%$ dos adolescentes declararam ter renda familiar média entre 1 e 3 salários mínimos, e 73 alunos (38,6\%) viviam em famílias com renda inferior a um salário mínimo, que à época da pesquisa significava $R \$ 545,00$.

Dos 189 questionários analisados, 46,0\% dos adolescentes moravam com ambos os pais, $15,3 \%$ com a mãee $14,8 \%$ com outros familiares. Quando perguntados sobre a iniciação sexual, $72 \%$ dos homens e $60,7 \%$ das meninas responderam afirmativamente.

Entre os 126 jovens que já haviam praticado relações sexuais, $89 \%$ tinham vida sexual ativa e $17 \%$ dos homens e $15,7 \%$ das adolescentes disseram não saber o que significava planejamento familiar.

Questionados sobre o conhecimento e a utilização de métodos contraceptivos, $10,1 \%$ dos adolescentes acreditam que não é possível engravidar na primeira relação sexual; $40,5 \%$ dos jovens referiram não utilizar nenhum método contraceptivo (Tabela 1). Não houve diferença estatisticamente significante entre a utilização de MAC para os sexos $(p=0,233)$, e a grande maioria dos adolescentes $(77,3 \%)$ utilizavam o preservativo masculino $(p=0,000001)$.
Tabela 1 - Distribuição dos adolescentes de 7as e $8^{\text {as s séries }}$ de uma escola pública quanto à utilização de métodos contraceptivos. Uberaba, 2011.

\begin{tabular}{|c|c|c|c|c|c|c|}
\hline \multirow{2}{*}{$\begin{array}{l}\text { Uso de métodos } \\
\text { contraceptivos }\end{array}$} & \multicolumn{2}{|c|}{ Masculino } & \multicolumn{2}{|c|}{ Feminino } & \multicolumn{2}{|r|}{ Total } \\
\hline & $\mathbf{N}$ & $\%$ & $\mathbf{N}$ & $\%$ & $\mathbf{N}$ & $\%$ \\
\hline \multicolumn{7}{|c|}{ Você faz uso de algum método contraceptivo?* } \\
\hline Sim & 26 & $36,1 \%$ & 23 & $42,6 \%$ & 49 & $38,9 \%$ \\
\hline Não & 27 & $37,5 \%$ & 24 & $44,4 \%$ & 51 & $40,5 \%$ \\
\hline \multirow[t]{2}{*}{ Às vezes } & 19 & $26,4 \%$ & 7 & $13,0 \%$ & 26 & $20,6 \%$ \\
\hline & 72 & $100,0 \%$ & 54 & $100,0 \%$ & 126 & $100,0 \%$ \\
\hline
\end{tabular}

Quais os métodos contraceptivos que você utiliza?**

\begin{tabular}{|c|c|c|c|c|c|c|}
\hline $\begin{array}{l}\text { Preservativo } \\
\text { masculino }\end{array}$ & 45 & $100,0 \%$ & 13 & $43,3 \%$ & 58 & $77,3 \%$ \\
\hline $\begin{array}{l}\text { Preservativo } \\
\text { feminino }\end{array}$ & 0 & $0,0 \%$ & 0 & $0,0 \%$ & 0 & 0,0 \\
\hline $\begin{array}{l}\text { Anticoncepcional } \\
\text { oral }\end{array}$ & 0 & $0,0 \%$ & 9 & $30,0 \%$ & 9 & 12,0 \\
\hline $\begin{array}{l}\text { Pílula do } \\
\text { dia seguinte }\end{array}$ & 0 & $0,0 \%$ & 6 & $20,0 \%$ & 6 & $8,0 \%$ \\
\hline \multirow[t]{2}{*}{ Injeção hormonal } & 0 & $0,0 \%$ & 2 & $6,7 \%$ & 2 & $2,7 \%$ \\
\hline & 45 & $100,0 \%$ & 30 & $100,0 \%$ & 75 & $100,0 \%$ \\
\hline
\end{tabular}

*A pergunta foi respondida apenas pelos jovens que relataram já ter praticado relações sexuais $(\mathrm{N}=126)$

**Respondida apenas pelos jovens que responderam "sim" ou "às vezes" na questão anterior $(\mathrm{N}=75)$

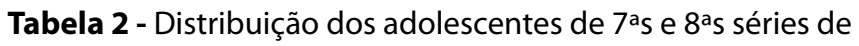
uma escola pública quanto ao consumo de bebidas alcóolicas. Uberaba, 2011.

\begin{tabular}{|c|c|c|c|c|c|c|}
\hline \multirow[t]{2}{*}{ Uso de Álcool } & \multicolumn{2}{|c|}{ Masculino } & \multicolumn{2}{|c|}{ Feminine } & \multicolumn{2}{|c|}{ Total } \\
\hline & $\mathbf{N}$ & $\%$ & $\mathbf{N}$ & $\%$ & $\mathbf{N}$ & $\%$ \\
\hline Sim & 66 & $66,0 \%$ & 49 & $55,1 \%$ & 115 & $60,8 \%$ \\
\hline Não & 19 & $19,0 \%$ & 28 & $31,5 \%$ & 47 & $24,9 \%$ \\
\hline Em branco & 15 & $15,0 \%$ & 12 & $13,5 \%$ & 27 & $14,3 \%$ \\
\hline \multicolumn{7}{|c|}{ Idade em que fez uso } \\
\hline 13 anos & 8 & $12,1 \%$ & 3 & $6,1 \%$ & $\mid 11$ & $9,6 \%$ \\
\hline 14 anos & 19 & $28,8 \%$ & 15 & $30,6 \%$ & 34 & $29,6 \%$ \\
\hline 15 anos & 23 & $34,8 \%$ & 24 & $49,0 \%$ & 47 & $40,9 \%$ \\
\hline 16 anos & 7 & $10,6 \%$ & 7 & $14,3 \%$ & 14 & $12,2 \%$ \\
\hline 17 anos & 9 & $13,6 \%$ & 0 & $0,0 \%$ & 9 & $7,8 \%$ \\
\hline \multicolumn{7}{|c|}{ Frequência de uso } \\
\hline Diária & 0 & $0,0 \%$ & 0 & $0,0 \%$ & 0 & $0,0 \%$ \\
\hline Semanal & 28 & $42,4 \%$ & 13 & $26,5 \%$ & 41 & $35,7 \%$ \\
\hline Mensal & 0 & $0,0 \%$ & 0 & $0,0 \%$ & 0 & $0,0 \%$ \\
\hline Eventualme & 21 & $31,8 \%$ & 17 & $34,7 \%$ & 38 & $33,0 \%$ \\
\hline Em branco & 17 & $25,8 \%$ & 19 & $38,8 \%$ & 36 & $31,3 \%$ \\
\hline
\end{tabular}


Tabela 3 - Distribuição dos adolescentes de 7as e 8as séries de uma escola pública quanto ao consumo de drogas ilícitas. Uberaba, 2011.

\begin{tabular}{|c|c|c|c|c|c|c|}
\hline \multirow{2}{*}{$\begin{array}{l}\text { Uso de Drogas } \\
\text { Ilícitas }\end{array}$} & \multicolumn{2}{|c|}{ Masculino } & \multicolumn{2}{|c|}{ Feminino } & \multicolumn{2}{|r|}{ Total } \\
\hline & $\mathbf{N}$ & $\%$ & $\mathbf{N}$ & $\%$ & $\mathbf{N}$ & $\%$ \\
\hline Sim & 38 & $38,0 \%$ & 23 & $25,8 \%$ & 61 & $32,3 \%$ \\
\hline Não & 43 & $43,0 \%$ & 44 & $49,4 \%$ & 87 & $46,0 \%$ \\
\hline Em branco & 19 & $19,0 \%$ & 22 & $24,7 \%$ & 41 & $21,7 \%$ \\
\hline \multicolumn{7}{|c|}{ Idade em que fez uso* } \\
\hline 13 anos & 2 & $5,3 \%$ & 0 & $0,0 \%$ & 2 & $3,0 \%$ \\
\hline 14 anos & 3 & $7,9 \%$ & 3 & $13,0 \%$ & 6 & $9,1 \%$ \\
\hline 15 anos & 25 & $65,8 \%$ & 19 & $82,6 \%$ & 44 & $66,7 \%$ \\
\hline 16 anos & 3 & $7,9 \%$ & 1 & $4,3 \%$ & 4 & $6,1 \%$ \\
\hline 17 anos & 5 & $13,2 \%$ & 0 & $0,0 \%$ & 5 & $7,6 \%$ \\
\hline \multicolumn{7}{|c|}{ Frequência de uso* } \\
\hline Diária & 0 & $0,0 \%$ & 0 & $0,0 \%$ & 0 & $0,0 \%$ \\
\hline Semanal & 3 & $7,9 \%$ & 0 & $0,0 \%$ & 3 & $4,9 \%$ \\
\hline Mensal & 10 & $26,3 \%$ & 5 & $21,7 \%$ & 15 & $24,6 \%$ \\
\hline Eventualmente & 6 & $15,8 \%$ & 3 & $13,0 \%$ & 9 & $14,8 \%$ \\
\hline Em branco & 19 & $50,0 \%$ & 15 & $65,2 \%$ & 34 & $55,7 \%$ \\
\hline
\end{tabular}

Sobreo conhecimento das diversas estratégias anticonceptivas, todos os alunos relataram conhecer o preservativo, $78 \%$ conheciam o anticoncepcional oral, $63 \%$ a pílula do dia seguinte e $51,3 \%$ o dispositivo intrauterino. Outros métodos não eram conhecidos por mais de $10 \%$ dos adolescentes.

Na Tabela 2, pode-se observar que a maioria dos adolescentes (66\% dos meninos e $55,1 \%$ das meninas) fazia uso de bebidas alcoólicas ( $p=0,0264$ ), cujo início se deu entre 14 e 15 anos ( $p=0,0335)$. As bebidas mais utilizadas eram a cerveja $(81,3 \%)$, seguida de Ice $(67,9 \%)$ e batidas $(54,2 \%)$. O consumo foi relatado acontecendo semanal ou eventualmente $(p=0,1227)$ e $31,3 \%$ deixaram a questão em branco.

Número expressivo de adolescentes (38\% dos meninos e $25,8 \%$ das meninas) já haviam ao menos experimentado uma droga ilícita ( $p=0,0630$ ). A maconha foi a droga mais utilizada por $47,9 \%$ dos adolescentes. 34,3\% assinalaram ter feito uso de cocaína, bem como mais de uma droga. Metade dos meninos (50,0\%) e 65,2\% das meninas não informaram qual a frequência de uso de droga.

\section{DISCUSSÃO}

A maioria dos estudantes avaliados era do sexo masculino, e boa parte dos adolescentes (38,6\%) pertencia a famílias com renda inferior a um salário mínimo, o que pode significar uma maior vulnerabilidade social e exposição a situações de risco, como abandono escolar, gravidez na adolescência, uso de álcool e drogas.

A iniciação sexual já havia ocorrido para $72 \%$ dos homens e $60,7 \%$ das mulheres. Estudo realizado em 2005, verificou que cerca da metade dos adolescentes iniciavam-se sexualmente aos 15 anos de idade, em sua maioria de forma não planejada $(72,7 \%)$ e dentro de casa $(86,1 \%)^{(10)}$. Pesquisa que envolveu
60.973 escolares de 1.453 escolas públicas e privadas, identificou que $43,7 \%$ dos meninos e $18,7 \%$ das meninas já haviam tido relação sexual, sendo que estudavam em escola pública $(33,1 \%)$, aos 16 anos de idade $(63,5 \%)$, utilizando o preservativo como método contraceptivo $(75,9 \%)^{(11)}$.

Entre a população estudada, 40,5\% referiram não utilizar nenhum método contraceptivo. Tal fato pode ser associado ao desconhecimento sobre contracepção e reprodução ${ }^{(12)}$, muitas vezes agravada pela falta de reflexão e consciência crítica perante o sexo, como é evidenciado no aumento dos casos de gravidez na adolescência, que tem sido relatada em alta prevalência nessa fase da vida ${ }^{(13)}$, que pode interferir no desenvolvimento e crescimento profissional dessas pessoas ${ }^{(12)}$, além de se constituir como fator de risco para a morbimortalidade materna e neonatal, expressa por complicações no parto, prematuridade e maiores riscos de aborto $^{(14)}$.

Com relação ao uso de álcool, observa-se que a maioria dos meninos (66\%) e das meninas $(55,1 \%)$ fazia uso mesmo que esporádico de álcool, tendo experimentado pela primeira vez aos 15 ou 16 anos. Estudo realizado com 499 adolescentes de Cuiabá/MT identificou que $45,2 \%$ dos meninos e $52,4 \%$ das meninas consomem bebidas alcoólicas, tendo iniciado o uso de bebida aos 15 anos $(49,1 \%)$, sendo a cerveja a mais comum ${ }^{(15)}$.

Entre os fatores que condicionam o consumo nessa idade, podem ser citados: contexto familiar e social, expectativas e crenças, preço acessível, disponibilidade comercial, facilidade de acesso e a utilização por pais ou familiares, que podem constituir um estágio de dependência na idade adulta ${ }^{(16)}$. Cabe ressaltar ainda que esses jovens estão cada vez mais expostos a estímulos para o consumo de álcool, através da mídia que em larga escala se utiliza de propagandas associando a bebida a situações de prazer e bem-estar.

Também se associa ao álcool uma série de situações desconfortáveis como aumento da violência intra e extrafamiliar, déficit e abandono escolar e outros. Estudo qualitativo realizado com 40 adolescentes entre 12 e 20 anos de Belém/PA, identificou um déficit no autocuidado dos adolescentes, que contribuiu para a exposição desses a situação de risco associada ao consumo abusivo de álcool, que ocorria predominantemente quando os jovens saiam em grupos de amigos ${ }^{(17)}$. Sugere-se a realização de ações educativas em saúde que despertem atitudes de autocuidado nos adolescentes a fim de mantê-los longe desses riscos.

Foram encontrados valores alarmantes quanto ao consumo de drogas na população estudada (38\% dos meninos e 25,8\% das meninas), sem dizer que esse número pode ser subestimado pelo montante de respostas em branco. No estudo de Jesus et $\mathrm{al}^{(15)}$, os valores encontrados foram de $20 \%$ e $18 \%$ respectivamente, cujo início se dava entre 15 e 16 anos, sua utilização era eventual em $49,1 \%$, e $28,9 \%$ já haviam feito uso de cocaína.

Estudo de revisão(18) associou fatores socioeconômicos e envolvimento familiar como fatores de risco para o consumo de álcool e outras drogas, além de apontar a maior utilização de cocaína e maconha, por serem as drogas mais acessíveis (menor custo e maior número de viciados), como apontaram os dados deste estudo. Ressalta-se a necessidade de suporte 
familiar e acompanhamento escolar adequado desses jovens para dirimir as situações de vulnerabilidade a que eles estão expostos, em especial na comunidade que fora objeto desta investigação. Outros fatores que podem ser associados ao consumo de drogas seriam a falta de lazer, as condições de vida dos jovens, dificuldades no envolvimento familiar (problemas de relacionamento, conflitos, ausência e falta de apoio dos pais), violência doméstica e aos amigos que usam droga.

Algumas iniciativas têm enfocado tais temas ao inserir estratégias de educação em saúde na escola voltadas para os adolescentes ${ }^{(1,19)}$ bem como para os professores ${ }^{(20)}$ desenvolvidas especialmente pelo profissional Enfermeiro, que possui como uma de suas principais atribuições a educação em saúde.

Nesse sentido, cabe pontuar a necessidade de maior articulação entre os setores saúde e educação para que se complementem, na busca de uma interdisciplinaridade capaz de enfrentar os desafios apresentados pelas situações de vulnerabilidade a que esses jovens estão expostos. Reconhece-se, ainda, a necessidade de investimento na capacitação dos profissionais da saúde e da educação para que os mesmos se sintam preparados e motivados a trabalhar com a temática da sexualidade na adolescência, o uso e abuso de substâncias químicas como drogas e álcool, entre outras, e na perspectiva da prevenção.

\section{CONSIDERAÇÕES FINAIS}

Osresultadosdesteestudorefletemanecessidadedeestratégias de educação em saúde direcionadas a adolescentes, observando a inserção de metodologias mais inclusivas, direcionadas e lúdicas, estimulando a reflexão crítica desses alunos acerca das situações de risco e vulnerabilidades relacionadas ao seu comportamento sexual, uso de bebidas alcóolicas e drogadição. Cabe considerar que esses resultados referentes a vulnerabilidades associadas à sexualidade e ao uso de drogas e álcool fazem parte de uma pesquisa que antecedeu a abordagem dessas temáticas em oficinas de prevenção, no desenvolvimento do projeto de extensão citado anteriormente.

Há que se priorizar ainda a capacitação do docente que estará em contato com esses alunos, fornecendo ao profissional de educação ferramentas para a abordagem dessas questões, entre outras; apoiado pela equipe das unidades de atenção primária à saúde de referência local.

Ressalta-se por fim, ao considerarem-se as carências sociais, econômicas e culturais da população estudada, a necessidade de maior articulação entre os setores de Segurança, Seguridade Social, Educação e Saúde, para trabalharem em conjunto na redução dos riscos de vulnerabilidade inerentes à complexidade da temática adolescente. Nesse sentido, fica apresentado o papel da Universidade que, ao trabalhar a pesquisa, permite conhecer a realidade local para propor estratégias interventivas no molde de extensão universitária.

\section{Referências}

1. Silveira RE, Reis NA, Santos AS, Borges MR, Soares SM. Oficinas com adolescentes na escola: uma estratégia de educação em saúde. Nursing (São Paulo). 2011;157(13):334-8. 2. Ferreira RA, Ferriani MGC, Mello DF, Carvalho IP, Cano MA, Oliveira LA. Spatial analysis of the social vulnerability of adolescent pregnancy. Cad Saúde Pública. 2012;28(2): 313-23.

3. Mendes SS, Moreira RMF, Martins CBG, Souza SPS, Matos KF. Saberes e atitudes dos adolescentes frente à contracepção. Rev Paul Pediatr. 2011;29(3):385-91 .

4. Alves $C A$, Brandão ER. Vulnerabilidades no uso de métodos contraceptivos entre adolescentes e jovens: interseçōes entre políticas públicas e atenção à saúde. Ciênc Saúde Coletiva. 2009;14(2):661-70.

5. Dias FLA, Silva KL, Vieira NFC, Pinheiro PNC, Maia CC. Riscos e vulnerabilidades relacionados à sexualidade na adolescência. Rev Enferm UERJ. 2010;18(3):456-61. 6. Koerich MS, Baggio MA, Backes MTS, Backes DS, Carvalho JN, Meirelles BHS, Erdman AL. Sexualidade, doenças sexualmente transmissíveis e contracepção: atuação da enfermagem com jovens de periferia. Rev Enferm UERJ. 2010;18(2):265-71. 7. Ruzany ML, Andrade CLT, Meirelles ZL, Moura EAF, Deusdará R, Rodrigues V, et al. Desinformação e vulnerabilidades com relação à sexualidade dos adolescentes e jovens da Reserva de Mamirauá, Amazonas - Brasil. Adolesc Saúde. 2010;7(2):41-9. 8. DATASUS. Departamento de Informática do Sistema Único de Saúde. Informaçōes sociodemográficas e de saúde [internet]. [citado em 2012 Mar 05]. Disponivel em: http://www.datasus.gov.br

9. Ministério da Saúde (BR). Departamento de Ações Programáticas e Estratégicas, Secretaria de Atenção à Saúde. Marco teórico e referencial: saúde sexual e saúde reprodutiva de adolescentes e jovens. Brasilia: Ministério da Saúde; 2006.

11. Malta DC, Silva MAI, Mello FCM, Monteiro RA, Porto DL, Sardinha MLV, et al. Saúde sexual dos adolescentes segundo a Pesquisa Nacional de Saúde dos Escolares. Rev Bras Epidemiol. 2011;14(Supl 1):147-56.

12. Villela WV, Doreto DT. Sobre a experiência sexual dos jovens. Cad Saúde Pública. 2006;22:2467-72.

13. Araújo MS, Costa LO. Comportamento sexual e contracepção de emergência entre adolescentes de escolas públicas de Pernambuco, Brasil. Cad Saúde Pública. 2009;25:551-62.

14. Brandão ER. Desafios da contracepção juvenil: interseções entre gênero, sexualidade e saúde. Ciênc Saúde Coletiva. 2009;14(4):1063-71.

15. Jesus FB, Lima FCA, Martins CBG, Matos KF, Souza SPS. Vulnerabilidade na adolescência: a experiência e expressão do adolescente. Rev Gaúcha Enferm. 2011;32(2):359-67.

16. Vieira DL, Ribeiro M, Romano M, Laranjeira RR. Álcool e adolescentes: estudo para implementar políticas municipais. Rev Saúde Pública. 2007;41(3):396-403. 17. Silva SED, Padilha MICS, Santos LMS. A enfermagem estimulando o autocuidado de adolescentes a partir das representações sociais desses sobre as bebidas alcoólicas. Enferm Foco. 2011;2(3):160-3.

18. Cavalcante MBPT, Alves MDS, Barroso MGT. Adolescência, álcool e drogas: uma revisão na perspectiva da promoção da saúde. Esc Anna Nery Rev Enfermg. 2008;12(3):555-9.

19. Koerich MS, Baggio MA, Backes MTS, Backes DS, Carvalho JN, Meirelles BHS, et al. Sexualidade, doenças sexualmente transmissiveis e contracepção: atuação da enfermagem com jovens de periferia. Rev Enferm UERJ, 2010; 18(2):265-71. 20. Silveira RE, Reis NA, Santos AS, Borges MR, Fonseca AS. Oficinas com professores: educação em saúde para o manejo com adolescentes. Acta Paul Enferm. (no prelo), 2012. 